Perspectiva Geográfica

ISSN 0123-3769 (Impreso) - 2500-8684 (En línea)

Vol. 21 N. ${ }^{\circ} 2$ de 2016

Julio - Diciembre

pp. 199-224

\title{
Exploración y explotación de petróleo: enfoque geográfico de los efectos ambientales en el Piedemonte llanero
}

\author{
Oil Exploration and Exploitation: A Geographic Focus on the \\ Environmental Effects in the Plain Foothills
}

Yesenia Vargas Tejedor ${ }^{1}$

Nohra León Rodríguez ${ }^{2}$

Para citar este artículo utilice el nombre completo así:

Vargas, Y. y León, N. (2016). Exploración y explotación de petróleo: enfoque geográfico de los efectos ambientales en el Piedemonte llanero. Perspectiva Geográfica, 21(2), 199-224. doi:10.19053/01233769.5795

\section{Resumen}

La investigación sobre los efectos ambientales de actividades económicas de gran incidencia para el país como lo son la exploración y la explotación de petróleo, abordadas desde la perspectiva de la geografía económica y la geografía ambiental (Eco geografía y Ecología política), permite desarrollar y utilizar métodos y técnicas para dimensionar de forma integral el conflicto existente en zonas de explotación de petróleo, como es el caso del Piedemonte llanero en el departamento de Casanare así como en otras áreas de similares características. En la identificación y el análisis de efectos ambientales se realizó una revisión crítica de los resultados obtenidos en dos componentes

1 Ingeniera Geóloga. Magíster en Geografía. Universidad Pedagógica y Tecnológica de Colombia. yesenia.vargastejedor@ uptc.edu.co

2 Doctora en Ciencias Económicas, Universidad Nacional de Colombia. Vicedecana Académica, Facultad de Ciencias Humanas, Universidad Nacional de Colombia - Sede Bogotá. nleonr@unal.edu.co 
(ambiental y económico), generando una matriz por década que permitió comparar los principales hitos reconocibles en la implementación de las políticas sectoriales y ambientales. Esto contrasta con variables como los cambios de cobertura, licencias ambientales, y otros resultados que lograron evidenciarse con la metodología empleada.

Palabras clave: ambiente, geografía, petróleo, política, ventaja comparativa.

\section{Abstract}

The research on the environmental effects of economic activity, of great impact for the country, such as the exploration and exploitation of oil approached from the economic geography and environmental geography perspectives (Eco geography and political ecology), allows to use and develop methods and techniques to dimension, comprehensively, the conflict in oil exploration areas such as the foothill plains in the department of Casanare, and other areas with similar characteristics. In the identification and analysis of environmental effects, a critical review of the results of two environmental and economic components was performed. Analyzing per decade and comparing the main recognizable landmarks in the implementation of sectorial and environmental policies, this contrasts with variables such as coverage changes, environmental licenses, and other results achieved which made conspicuous the used methodology.

Keywords: environment, geography, oil, politics, comparative advantage. 


\section{Introducción}

La existencia o ausencia de recursos naturales renovables y no renovables condicionan las actividades económicas en una región, en especial cuando hay fuerte presencia de un modelo extractivista de recursos mineros y energéticos. Kerschner y Arto (como se citó en Velte, 2011) hacen referencia a la existencia de tensiones en el mercado mundial de petróleo y gas debido al desajuste entre oferta y demanda del recurso energético.

Partiendo de los debates entre la geografía económica y ambiental, y su dificultad para integrarse en el estudio del ambiente (que a la vez constituye una necesidad cuando se trata de analizar efectos ambientales generados por una actividad económica), se plantea la hipótesis de que los efectos ambientales generados por la exploración $\mathrm{y}$ explotación de petróleo durante el período 1970-2014 en el corredor petrolero de Piedemonte, no fueron dimensionados por las políticas sectoriales y ambientales del país.

El objetivo general de la investigación fue analizar los efectos ambientales de la exploración y explotación de petróleo en el Piedemonte llanero.
Dentro de sus objetivos específicos: (a) determinar desde la geografía económica las razones que han definido la distribución espacial de bloques de exploración y explotación de hidrocarburos en el departamento de Casanare en el período comprendido entre 1970 a 2014. (b) Establecer por medio de un enfoque integral entre geografía económica y ambiental los efectos ambientales de las políticas ambientales y sectoriales en el escenario de estudio definido.

\section{Marco teórico}

Los aportes teóricos de la geografía considerados incluyeron las perspectivas teóricas del ambiente en la geografía moderna, la relación entre ambiente y desarrollo así como entre conflictos ambientales y la explotación de recursos naturales, destacando que el desarrollo teórico en este componente no pretende debatir la existencia de una disciplina híbrida. Por otro lado, la investigación parte de considerar a la geografía ambiental como un énfasis o una perspectiva requerida en la geografía (Bocco y Urquijo, 2013).

Es necesario reconocer que el ambiente no se trata sólo del lugar en el que se desarrolla la vida sino que además de englobar a los seres vi- 
vos, comprende objetos: agua, suelo, aire y las relaciones entre ellos, así como elementos tan intangibles como la cultura (Trinca, 2010). Por lo demás, su transformación responde a negociaciones entre actores sociales en distintos niveles de poder: "es un principio social, que se distingue de otros, como: ecosistema, bioma o geosistema, que hacen una valoración del estado de la naturaleza a partir de posturas analíticas fundamentalmente biofísicas" (Bocco y Urquijo, 2013, p. 84).

En este sentido, Sabatini (1997) indica que la diferencia entre conflicto ambiental y conflicto socioambiental es muy poca y por lo tanto es necesario eliminar esta barrera, ya que de acuerdo a Fontaine (2004), la distinción entre estos conflictos es discutible pues no existe "conflicto ambiental" sin dimensión social.

La geografía económica también ha permitido analizar diferentes efectos del desarrollo de actividades económicas que giran alrededor de la explotación de recursos naturales no renovables y que a su vez obedecen a sistemas económicos cambiantes e imperantes. Según Barnes (2001), es una disciplina diversa y con capacidad para una amplia variedad de perspecti- vas, algunas veces contradictorias en los intereses de fondo; un ejemplo de esto es el análisis de los recursos naturales.

Gunton (2003) por su lado llama la atención sobre dos corrientes, la primera, orientada a que los recursos naturales constituyen una barrera para el desarrollo regional, pues genera una fuerte dependencia económica; la segunda, de los teóricos de la ventaja comparativa, quienes argumentan que los recursos pueden acelerar el desarrollo regional.

En los debates actuales alrededor de la geografía se encuentra la necesidad de avanzar en una mejor integración de la geografía económica a la hora de analizar las dinámicas económicas a diferentes escalas, y cómo estas afectan el ambiente. Es así como Bridge (2008) analiza el surgimiento de la Geografía Económica del Ambiente (GEE) y la considera como un llamado a la acción colectiva, donde la geografía económica tiene la oportunidad de realizar un examen continuado de un cúmulo de fenómenos del ambiente contemporáneo.

Heidkamp (2008) define a la geografía económica del ambiente (GEE) como un sub-campo de la 
geografía económica que ha sido recientemente objeto de discusión debido a su estatus actual y proyección a futuro. Hace énfasis en que la geografía económica, a pesar del enfoque inherentemente interdisciplinario de la geografía y la larga tradición de investigación de las relaciones hombre-ambiente, ha ignorado por un periodo bastante largo y en gran medida el ambiente, sin ir más allá de su tratamiento como un factor más o menos pasivo que condiciona la localización de varias actividades económicas o insumos para la producción de bienes y servicios.

De acuerdo a lo anterior, la geografía podría tener un impacto significativo mediante la documentación de las diversas formas en que lo "económico" está incrustado en los espacios "extra-económicos", además de los procesos que con- vencionalmente se han etiquetado como del ambiente, y cómo el carácter de ambiente integrado en la actividad económica es importante para el funcionamiento de los sistemas económicos y los medios de vida que dependen de ellos (Bridge, 2008).

\section{Descripción del área de estudio}

El área de estudio se localiza en el departamento de Casanare. La unidad espacial de referencia es un área de 338.158,54 ha, en la zona del Piedemonte llanero con cubrimiento parcial de los municipios de Yopal, Aguazul y Tauramena, reconocida como el "Corredor petrolero de Piedemonte", y donde se localizan las principales áreas y empresas de explotación de petróleo en el departamento. En la Figura 1 se indica la localización general y específica del área de estudio.

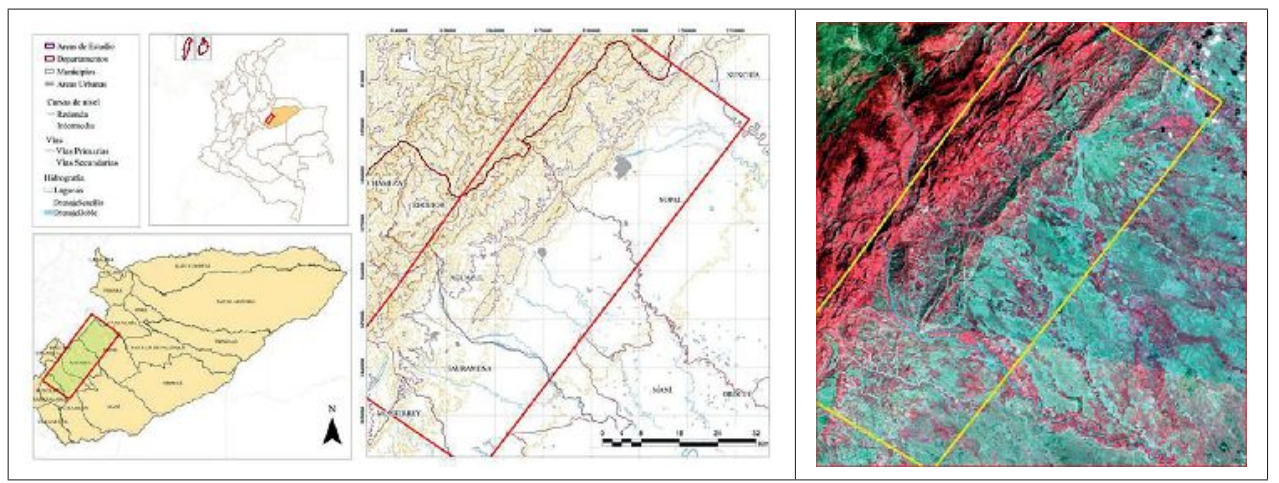

Figura 1. Localización del área de Estudio

Fuente: Elaboración propia (Información base IGAC, 2011. Imagen Landsat ETM Earth Explorer-USGS). 


\section{Metodología}

La perspectiva de la geografía ambiental permitió identificar y analizar aspectos críticos de las relaciones entre el hombre y la naturaleza (o del hombre como parte de los ecosistemas), además de analizar cómo los cambios ambientales pueden estar correlacionados con procesos sociales y políticos a diversas escalas.

La investigación también incorporó el análisis geo-histórico, de gran importancia para los análisis intertemporales durante el período definido, principalmente en la utilización de la denominada cartografía histórica que tiene por objeto "la reconstrucción de la estructura y dinámica espacial de un lugar y período determinado, en la cual se representan las acciones sociales derivadas de la realidad, permitiendo reconstruir los procesos espaciales desde lo sincrónico (espacio) y lo diacrónico (tiempo)" (Aponte, 2006).

La metodología (Figura 2) desarrollada para el análisis de efectos ambientales de la exploración y explotación de petróleo en el Piedemonte llanero, específicamente en el corredor Yopal, Aguazul, Tauramena, se explica a continuación.

\section{a. Geografía económica}

El desarrollo metodológico a partir de la geografía económica integró los siguientes componentes de acuerdo a sus aportes teóricos en las teorías de la localización, la relación entre recursos naturales y el desarrollo regional, así como las propuestas de la geografía económica del ambiente:

Definición de periodos de análisis. Recopilación y agrupación de información y análisis de acuerdo a los períodos de gobierno, incluyendo el contexto internacional de reservas probadas y producción.

Revisión y análisis de planes nacionales de desarrollo (PND). De once períodos de gobierno desde el año 1970 hasta el año 2014, haciendo énfasis en las estrategias definidas en materia de exploración y explotación de petróleo.

Procesamiento y análisis de los datos de reservas probadas y explotación de petróleo. Las técnicas cuantitativas fueron empleadas para poder identifi- 
car patrones de comportamiento de algunos fenómenos. En este caso se identificaron los datos de reservas probadas y de explotación de petróleo disponibles para el país (BP, 2015), así como los registros históricos de la denominada cartografía de tierras de la Agencia Nacional de Hidrocarburos (ANH) e información del Banco de Información Petrolera de Colombia (EPIS). Es importante indicar que los datos tienen la misma ventana de tiempo de los PND.
Teniendo en cuenta que las dinámicas espaciales de la actividad económica asociada al petróleo no pueden ser estudiadas fuera de un contexto mundial, dicho análisis consideró la situación de Colombia en la distribución espacial de las reservas mundiales de petróleo, producción y consumo, para finalmente analizar la distribución espacial de las áreas asignadas para exploración y explotación de hidrocarburos en esta región.

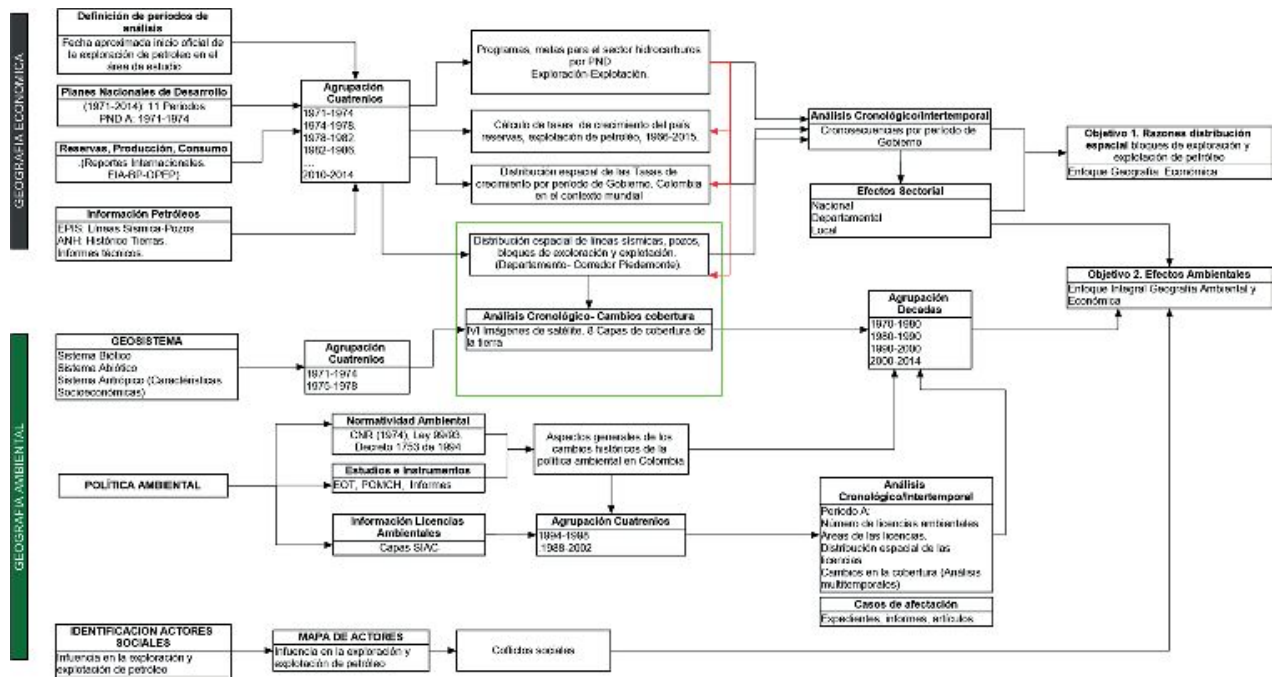

Figura 2. Diagrama general de la metodología aplicada para el desarrollo del proyecto de investigación

Fuente: Elaboración propia.

Análisis intertemporal. Cronosecuencia de la información de tierras (áreas de exploración y explotación de petróleo) para el país, el departamento de Casanare, las líneas de prospección sísmica y pozos en el "Corredor petrolero del Piedemonte" des- 
de 1974 a 2015; considerando el año de inicio de acuerdo a la fecha del primer contrato para exploración de petróleo firmado en el área de estudio definida.

\section{b. Geografía ambiental}

Conforme a los aportes teóricos de la geografía ambiental, inicialmente se realizó una caracterización del área de estudio, considerando tres componentes generales como son:

Geosistema. La descripción fue realizada teniendo en cuenta los subsistemas abiótico (Clima, geomorfología, climatología, hidrografía, orografía), biótico (vegetación, flora, fauna) y antrópico (densidad de población, actividades económicas, usos del suelo, y otras características socioeconómicas del área). Partiendo de los estudios previos desarrollados por entidades del orden internacional, nacional y local, entre ellas: World Wild Foundation (WWF), IDEAM, IGAC, Corpoboyacá, Corporinoquia, la Gobernación del departamento de Casanare, las Alcaldías municipales de Aguazul, Tauramena, Yopal, entre otros.
Actores sociales. La información de referencia fueron estudios realizados por la Universidad Nacional (2013) y Devia (2011), en donde se identificaron seis grupos de actores que han influenciado la actividad de exploración y explotación de petróleo en el área de estudio: (1) actores internacionales, (2) actores estatales del nivel local, regional y nacional, (3) comunidad en general, (4) empresas petroleras, (5) empresas asociadas a la actividad petrolera y (6) grupos armados legales e ilegales.

Politica Ambiental. Para identificar algunos de los efectos de estas políticas, se analizaron y contrastaron los tres siguientes aspectos: (a) las generalidades de la política ambiental global y la evolución de las políticas ambientales en Colombia; (b) la caracterización del área de estudio, identificación de los actores sociales de influencia en la exploración y explotación de petróleo; y (c) la evaluación espacial cuantitativa de los cambios en la cobertura de la tierra.

La integración de estos componentes permitió adicionar y com- 
plementar al enfoque puramente biofísico con el que muchas veces se asocia a la geografía ambiental (Bocco y Urquijo, 2013). Con fundamento en aportes teóricos de la ecogeografía y la ecología política, que indican cómo los cambios ambientales están sumamente influenciados por procesos sociales y políticos a diversas escalas, se realizó la recopilación de información y cartografía disponible en el Sistema de Información Ambiental de Colombia (SIAC), sobre áreas protegidas y licencias ambientales otorgadas para el sector de hidrocarburos en el departamento. También se recopiló información complementaria de la Corporinoquia pues constituye una parte de los productos de la política ambiental del país, dentro del corredor petrolero de Piedemonte.

\section{c. Análisis integral}

Para establecer los efectos ambientales de las políticas económicas y sectoriales (asociadas a la exploración y explotación de petróleo en el área de estudio), a través de un enfoque integral entre geografía económica y ambiental, se integraron los resultados obtenidos en los componentes ambiental y económico (sectorial). Se emplearon, además, diferentes recursos para los análisis de cambios que permitieran evidenciar cuales fueron los comportamientos más críticos en los escenarios pasados, con el fin de validar la hipótesis propuesta.

Se integró la información existente sobre áreas protegidas de carácter local, regional y nacional en el área de estudio definida, así como fechas, tipos de declaración y cronosecuencias de información disponible sobre licencias ambientales otorgadas para el desarrollo de la actividad petrolera (exploración y explotación). Debido a la importancia de los cambios en la vegetación se generaron mapas y matrices de transición para identificar cambios en la cobertura, (ganancias, pérdidas y permanencia de la misma de una fecha a otra), pues son los principales motores de cambio global por sus efectos en el clima, los procesos ecosistémicos, los ciclos biogeoquímicos y la biodiversidad de los ecosistemas naturales (Vitousek, et al., 1997).

La información mencionada anteriormente fue contrastada con información espacial asociada a la exploración y explotación de petróleo. Por su parte, la comparación de cronosecuencia fue contrastada 
con problemas ambientales identificados en el área de instrumentos de planificación ambiental y territorial y reportes de la comunidad, además de información secundaria que hace referencia a las afectaciones directas ocasionadas por la actividad petrolera en el área de estudio. Esta información proviene de la Autoridad Nacional de Licencias Ambientales (ANLA), la Agencia Nacional de Hidrocarburos (ANH), el Sistema Nacional de Áreas protegidas (SINAP), Sistema de Información Ambiental de Colombia (SIAC), planes de ordenación y manejo de cuencas (CORPORINOQUIA), registros históricos en medios, entre otros.

Para la identificación de efectos ambientales se realizó una revisión crítica de los resultados obtenidos en los componentes ambiental y económico, generando una matriz por década (años setenta, ochenta, noventa y el período del año 2000 al año 2015) y comparando los principales hitos reconocibles en la implementación de las políticas sectoriales y ambientales. Contrastando con efectos en el país y en el departamento, así como otros resultados que lograron evidenciarse con la metodología empleada.

\section{Resultados y discusión}

Distribución espacial de bloques de exploración y explotación de hidrocarburos en el departamento de Casanare en el período comprendido entre el año 1970 al año 2014

Como parte del análisis sobre los efectos de los PND del gobierno nacional durante los últimos once años, en la exploración y explotación de petróleo, se evidenció que en la última década a nivel nacional se incrementó notablemente la oferta de áreas para el interés del sector de los hidrocarburos. Sin embargo, en el departamento de Casanare se intensifica incluso desde años atrás (ver Figura 3).

La Figura 4 indica que las mayores tasas de crecimiento en el país, para reservas probadas, se presentaron en los periodos de 19821994, y el último período 2010 2014 coincidente con los períodos de gobierno de Belisario Betancur, Virgilio Barco, César Gaviria y Juan Manuel Santos. Sin embargo, llama la atención que en los períodos 1994-1998 y 2006-2010, se presenta un incremento considerable de la tasa de crecimiento en explotación de petróleo aun cuando no hay un crecimiento positivo en 
las reservas probadas. Esto indica que no se tuvo éxito en el hallazgo de nuevos yacimientos pese a las metas de exploración definidas en estos períodos. De igual forma, se evidencia que los mayores incrementos en la tasa de crecimiento de reservas probadas es el período 1982-1994. En la década de los noventa el fuerte incremento de la tasa de crecimiento, coincide especialmente con el descubrimiento y la oficialización de los campos $\mathrm{Cu}$ siana y Cupiagua en Casanare.

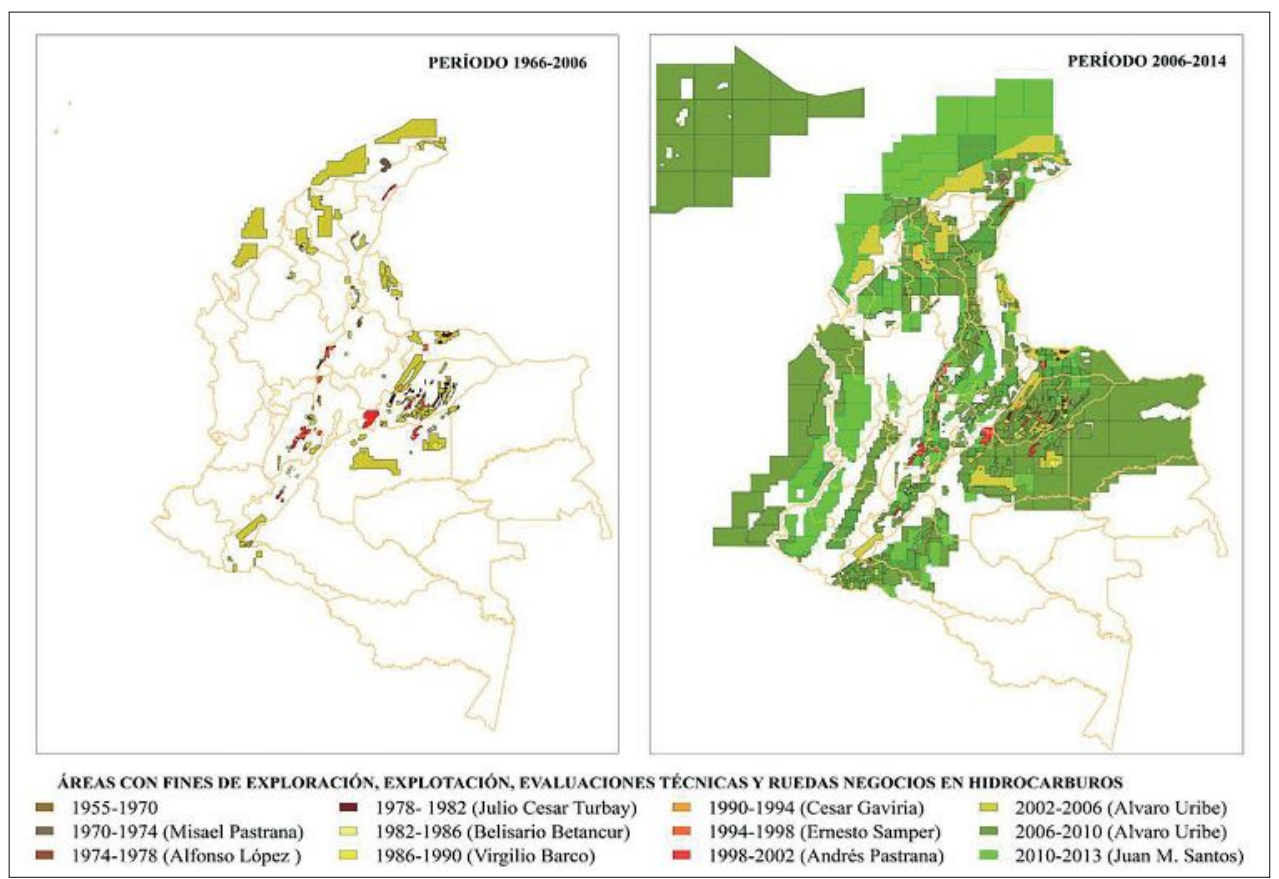

Figura 3. Evolución de la distribución espacial de los bloques de exploración y explotación de petróleo a nivel nacional durante los periodos de gobiernos de 1998 a 2014

Fuente: Elaboración propia con datos de la ANH (2014). 

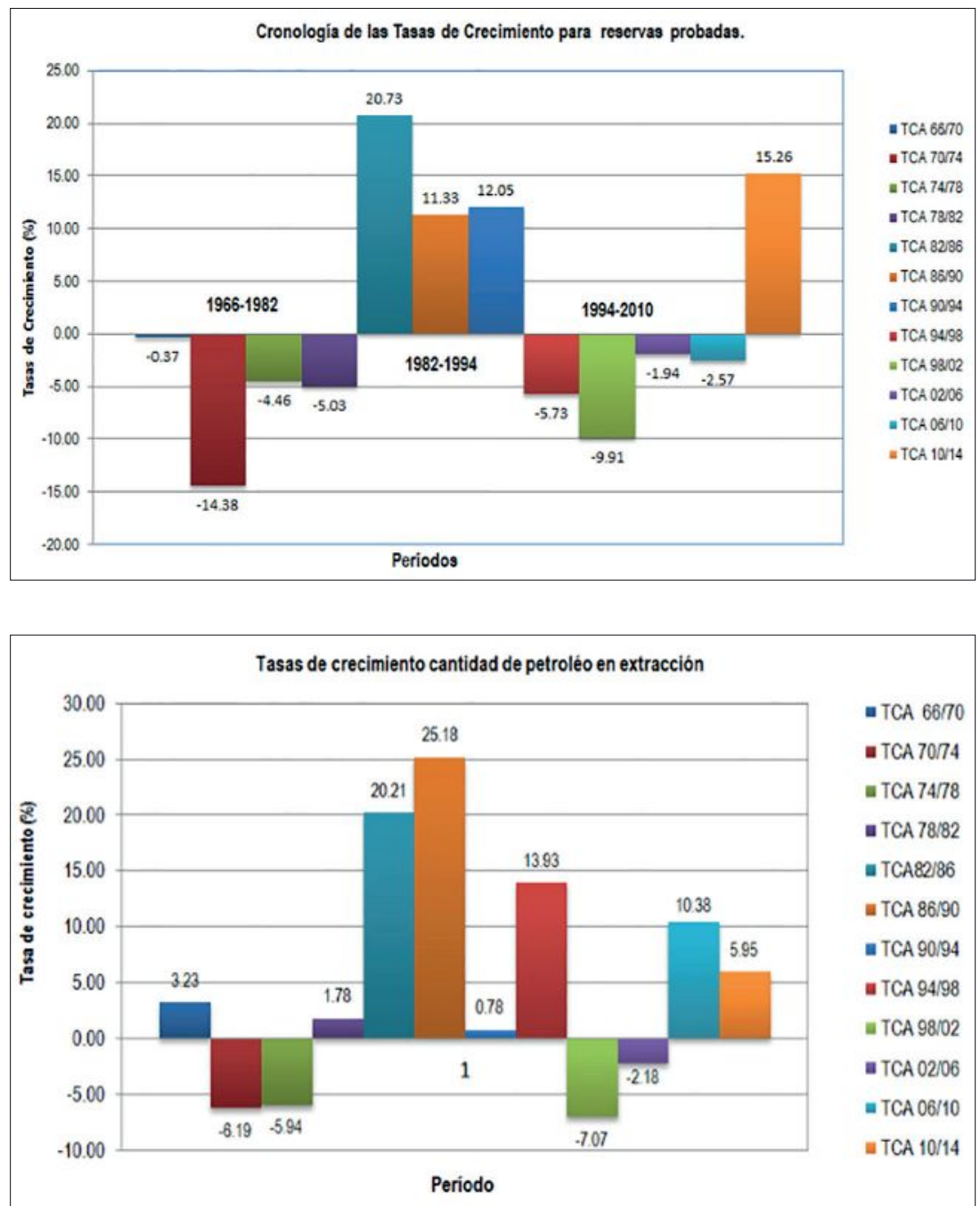

Figura 4. Cronología de las tasas de crecimiento de reservas probadas y explotación de petróleo en Colombia, período (1966-2014). (Primer gráfico) Tasa de crecimiento Reservas probadas. (Segundo gráfico) Tasa de crecimiento - Cantidad de petróleo en extracción

Fuente: Elaboración propia.

Además de la distribución espa- dades asociadas al sector de hidrocial de las áreas de interés dentro carburos se convierten en fuerzas del departamento de Casanare, es centrípetas, que han atraído capitaimportante indicar cómo las activi- les extranjeros representados por la 
presencia de empresas con diferentes países de origen, donde todos confluyen en una misma área geográfica. Algunas de las empresas son grandes trasnacionales como British Petroleum (BP), Petrobras, y otras de menor capital que actúan solas o en uniones de empresas extranjeras y nacionales, de carácter público o privado $\mathrm{y}$ en forma de alianzas público-privadas. En esta identificación y posterior espacialización, llama la atención que además del Reino Unido, Canadá, Estados Unidos y España, se encuentran empresas que tienen sus orígenes en países como Australia, Barbados, China, India, Rusia, Corea, además de origen suramericano como Brasil, Perú, Ecuador, Argentina, Chile y México.

Como se observa en la Figura 5, la mayoría de áreas fueron otorgadas en el departamento de Casanare durante el período 2006-2010, pero la dinámica se intensificó a partir del período 1998-2002. No obstante, es claro, de acuerdo a las metas del PND de estos períodos de gobierno, que el incremento significativo de áreas es consecuencia de la planeación y las metas definidas, también, a partir de la planeación de gobiernos anteriores.

Otra de las características en la distribución espacial de los bloques, es que la mayoría de ellos fueron administrados u operados por empresas privadas multinacionales extranjeras. No obstante, en el caso del corredor petrolero piedemonte al año 2015 es operado por la unión de empresas público-privadas. Esto se debe a la finalización del contrato de asociación con British Petroleum (BP), que permitió dar lugar al control de Ecopetrol a partir del año 2010. Es importante indicar que BP fue la principal operadora con presencia en el área por más de veinte años.

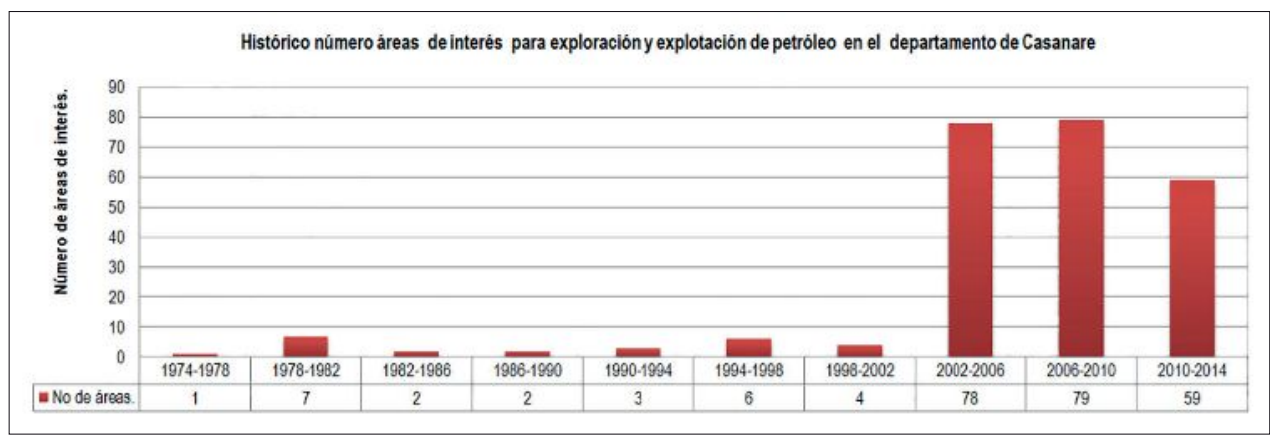




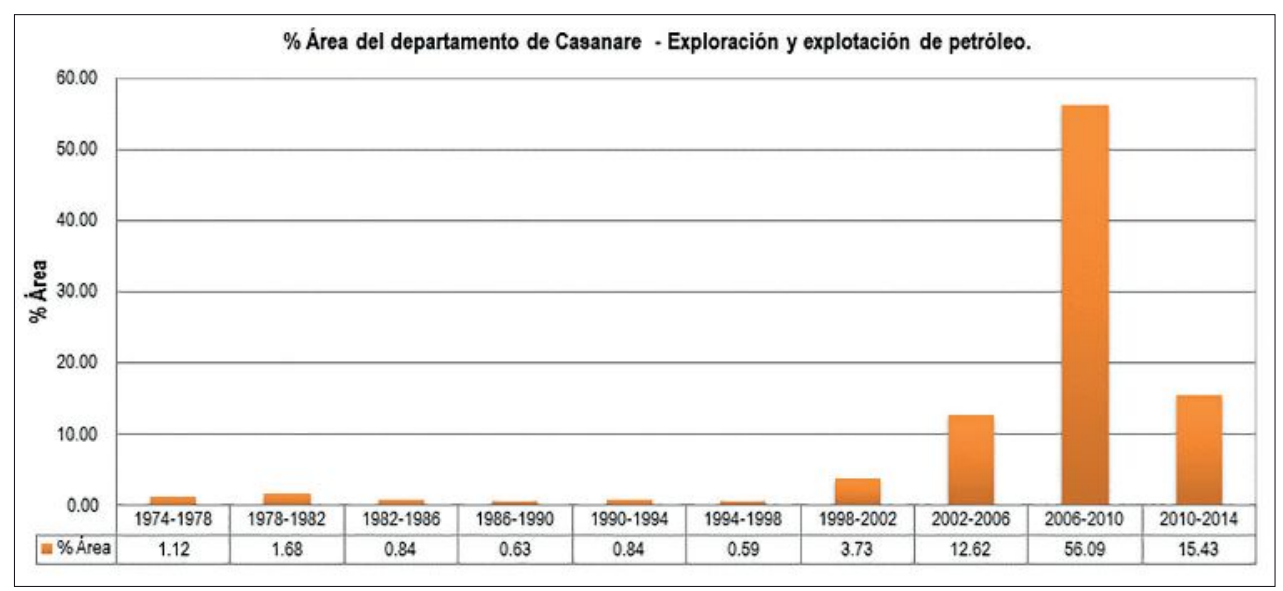

Figura 5. Cronología del número de áreas otorgadas por el gobierno nacional para interés del sector en materia de exploración y explotación de petróleo (1974-2014) en el departamento de Casanare. (Primer gráfico) Número de áreas. (Segundo gráfico) Porcentaje con respecto al área del departamento

Fuente: Elaboración propia.

\section{Efectos de la implementación de políticas ambientales}

El análisis general de la evolución de la política ambiental en Colombia (Figura 6), sugiere que estas políticas no se han logrado integrar con las políticas sectoriales debido a la ausencia de una visión integral del territorio, sobre todo en áreas en donde se desarrollan actividades de explotación de recursos naturales no renovables, y donde la identificación de actores sociales muestra que son varios los intereses sobre estas áreas. En otras palabras, cuestionar la dimensión territorial de las políticas ambientales, como afirma Rebotier:
Son obvios los límites de aquellas herramientas reglamentarias y de gestión que demuestran pocas consideraciones para los contextos locales (para los territorios, a pesar de lo pregonado), para la complejidad de los factores involucrados y sus interacciones, y para las dimensiones sociales y humanas de los riesgos. (Rebotier, 2013, p. 6)

\section{Análisis de la distribución espacial de las licencias ambientales}

A continuación se presentan algunas consideraciones sobre los análisis realizados por períodos de gobierno (a partir de la década de los 
noventa), así como de los resultados de la distribución espacial de las licencias otorgadas con fines de exploración y explotación (Figura 7).

Durante el período 2006-2010, correspondiente al segundo gobierno de Álvaro Uribe Vélez, se otorgaron el mayor número de licencias ambientales en el departamento de Casanare (más de 350), descendiendo durante el siguiente perío- do de gobierno. Sin embargo, esto no quiere decir que el descenso se deba a la disminución de estas actividades en el departamento, particularmente teniendo en cuenta que la exploración sísmica no requiere de licenciamiento ambiental. Lo anterior también obedece a que de la gran cantidad de área otorgada para exploración y explotación durante el 2006-2010, solo el 40\% tiene licencia ambiental.

\begin{tabular}{|c|c|c|c|}
\hline Clásica. S XIX- 1936 & $\begin{array}{c}\text { Productivista } \\
\text { 1936-1991 }\end{array}$ & $\begin{array}{c}\text { Desarrollo Sostenible } \\
1991-2002\end{array}$ & \\
\hline \multicolumn{4}{|c|}{ Etapas de las politicas ambientales en Colombia. (Guhl Nannetti \& Leyva, 2015) } \\
\hline & $\begin{array}{l}\text { Conservacionista. } \\
\text { Década del } 70 \text { y } 80\end{array}$ & $\begin{array}{c}\text { Desarrollo Sostenible } \\
\text { Década de los } 90 \text {. }\end{array}$ & $\begin{array}{c}\text { Privatización de la } \\
\text { gestión ambiental. } 2002 \text { - } \\
2014\end{array}$ \\
\hline \multicolumn{4}{|c|}{$\begin{array}{l}\text { La Conferencia de Estocolmo (1972) influyo para que en } \\
\text { Colombia se promulgara el Código de los Recursos Naturales y } \\
\text { Protección delAmbiente (1974). }\end{array}$} \\
\hline \multicolumn{4}{|c|}{$\begin{array}{l}\text { Ley } 99 \text { de } 93 \text {. Creación del SINA. } \\
\text { Decreto } 1753 \text { de } 1994 \text { reglamentación de licencias ambientales. } \\
\text { Objetivos orientados, hacia el desarrollo sostenible en articulación } \\
\text { con la cumbre de Río de } 1992 \text {. Rodríguez (2002) y Mufioz (2011) } \\
\text { Inclusión de lo ambiental en la constitución politica de } 1991\end{array}$} \\
\hline Creación del & nisterio del medioambient & \multicolumn{2}{|c|}{$\begin{array}{l}\text { Creación del MAVDT, } 2003 \text {. } \\
\text { Pérdida de posición politica de lo ambiental. } \\
\text { Desarticulación del Sistema Nacional Ambiental, SINA. } \\
\text { Creación de áreas protegidas. } \\
\text { Debilitamiento del licenciamiento y los instrumentos de gestión }\end{array}$} \\
\hline
\end{tabular}

Figura 6. Tipos de política ambiental en Colombia (Tobasura, 2006), adaptado de Roth Deubel, Andre Noel, 2004. Seguido de etapas de las políticas ambientales en Colombia (Guhl Nannetti y Leyva, 2015)

Fuente: Elaboración propia.

Localmente se observa que en casi o en la gran mayoría de las áreas que han sido identificadas como áreas de protección y conservación regional y local, se han otorgado licencias ambientales para desarrollar actividades asociadas al petróleo especialmente en las áreas de reserva de la sociedad civil, específicamente en el área mues- 
tra definida dentro del Piedemonte llanero, con excepción del Parque Natural de San Miguel de Farallones y La Tablona.

Por otra parte, al identificar las empresas solicitantes de las licencias ambientales que implican la utilización de varios servicios ambientales, se observa que en su gran mayoría son empresas trasnacionales que tienen origen en diferentes países. Vale resaltar que estas empresas tienden a considerar los recursos naturales más como un capital natural que como un patrimonio natural:

Considerando la naturaleza como factor productivo y su preservación como una forma de inversión ligada a los intereses particulares. Bajo esta idea se imponen esos criterios sobre la soberanía de los Estados, mientras sus habitantes sufren el desperdicio y usurpación de sus recursos, especialmente el agua. (Ramírez y Yepes, 2011, p. 151)

De igual forma, es necesario considerar que aunque existe una gran cantidad de áreas y pozos que cuentan con licencia ambiental (algunas de ellas ya archivadas o en seguimiento). Y aun cuando exis- ten o implicaron medidas de compensación, es importante cuestionar hasta qué punto compensarán el pasivo ambiental dejado en el departamento, que como reflejan los análisis de la distribución de bloques de exploración y explotación, así como el de licencias ambientales, este es un territorio que ha sido sometido a una fuerte presión especialmente desde la década de los noventa, intensificándose en la primera década del siglo XXI.

\section{Principales cambios en la cober- tura}

De acuerdo al análisis de cronosecuencias de la cobertura de la tierra (Figura 8), es claro que existen tres momentos que han marcado los cambios de cobertura en la zona de muestra del Piedemonte, tanto en el corredor petrolero como en zonas aledañas. Como todo análisis cronológico, se parte de un punto base, que para este caso fue el año 1972. Ahora, teniendo en cuenta la caracterización socioeconómica del área de estudio realizada anteriormente, se podría considerar que los cambios durante las décadas de los setenta y los ochenta obedecen a una expansión de la frontera agrícola favorecida por las campañas de prospección sísmica que facili- 

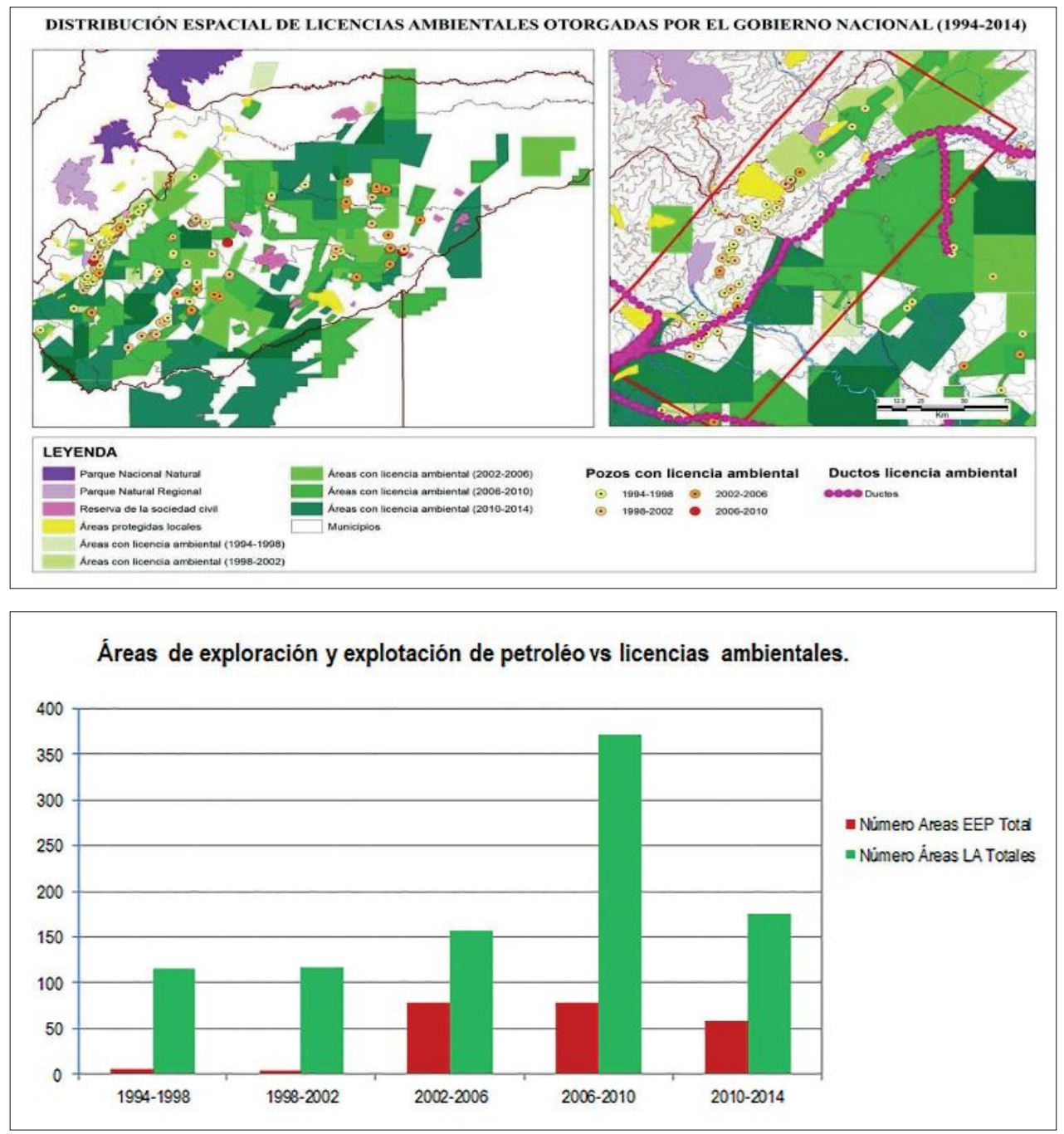

Figura 7. (Primer gráfico) Distribución espacial de las licencias ambientales otorgadas por el gobierno nacional para realizar actividades asociadas a la exploración y explotación de hidrocarburos, agrupados en los períodos 1994-1998, 1998-2002, 20022006, 2010-2014 (Izq. Distribución en el departamento. Der. Distribución dentro del área de estudio). (Segundo gráfico) Relación del número y áreas para exploración y explotación de petróleo en el departamento de Casanare con respecto al número y áreas de licenciamiento ambiental del sector hidrocarburos otorgadas en el departamento de Casanare

Fuente: Elaboración propia con base a los datos del sistema de información ambiental de Colombia. 
taron procesos masivos de colonización. Claramente se indica que durante la década de los setenta y los ochenta la economía se sustentaba en las actividades agropecuarias, y que se encontraba en estado de expansión teniendo en cuenta la calidad del clima y los suelos del
Piedemonte, donde la cobertura boscosa es la que presenta una mayor pérdida, dando paso a los territorios agrícolas (específicamente en el área se realizaron 275 líneas de prospección. 99 en los años setenta; 173 entre el los años 19801986 y 32 entre 1986-1990).

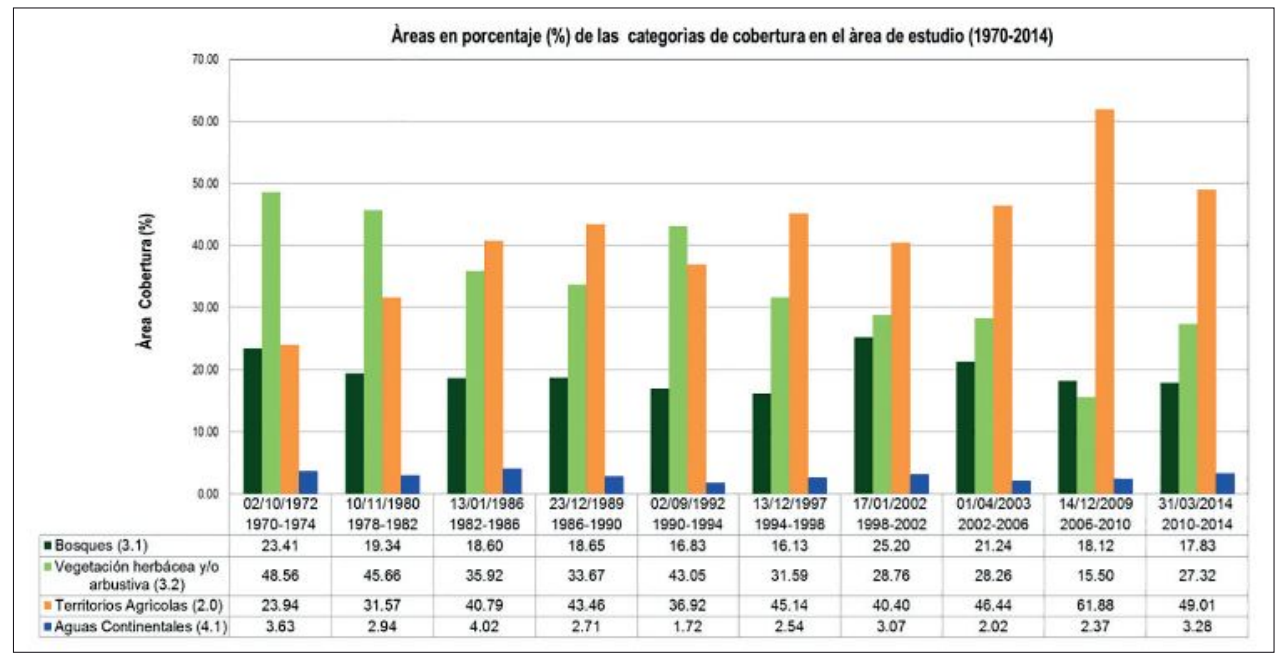

Figura 8. Serie de tiempo de los porcentajes de las áreas de cobertura en el área de estudio (1970-2014). En cada uno de los períodos se indica la fecha de la imagen utilizada

Fuente: Elaboración propia.

En el segundo momento, durante la década de los noventa, se continúan registrando pérdidas de áreas de cobertura boscosa y territorios agrícolas que se convierten en zonas de vegetación herbácea y arbustiva. Se realizaron durante esta época 161 líneas de prospección sísmica. En esta década se descubren los yacimientos de petróleo en
Cusiana y Cupiagua (en los años 1991 y 1993 respectivamente). Las actividades económicas en la zona desde finales de los años ochenta y durante la década de los noventa giraron en torno a la exploración y explotación del petróleo en el Piedemonte, reduciendo la actividad agropecuaria. En este sentido, se considera que las transforma- 
ciones de la cobertura de la tierra estuvieron directamente ligadas a la realización de las campañas de prospección sísmica y a la adecuación del Piedemonte para el desarrollo de las zonas de explotación y la construcción de los Centros de Producción y Facilidades (CPF).

En el tercer momento, entre el año 2000 y el año 2014, se observa una clara disminución de las áreas de coberturas de bosques y de la vegetación herbácea y arbustiva. De acuerdo a los análisis de cambios netos para las dos categorías, sus áreas de pérdida se convirtieron en territorios agrícolas. Teniendo en cuenta que de acuerdo a los históricos de los precios del petróleo, durante el período 2002-2014, el barril alcanzó su valor más alto, y aunque ya en consolidación del corredor petrolero se realizaron más campañas de prospección sísmica (46 líneas), perforación de pozos exploratorios y de desarrollo (y a su vez fue el período en el que más se otorgaron licencias ambientales), se considera en consecuencia que las transformaciones de las coberturas mencionadas son producto de los cambios en las formas de licenciamiento ambiental, y en el seguimiento y efectividad de planes de manejo ambiental que, como han expresado los diferentes autores citados en este componente, han llevado a la flexibilización de estos instrumentos sin el suficiente rigor técnico tanto en los estudios como en el seguimiento de los planes de manejo.

\section{Afectaciones del modelo petrolero} en el piedemonte llanero. Período 1970-2014

Es posible deducir que en el Piedemonte llanero se han evidenciado similares conflictos e impactos ambientales ocasionados por el desarrollo de la actividad petrolera. En otros países de la región también se reflejan dichos fenómenos (entre los que se encuentran los cambios de las coberturas y por ende afectaciones en los ecosistemas), que no solo se limitan a los bloques de exploración, sino también sobre el efecto de borde y más allá de los perímetros en terrenos que claramente definen las empresas (Bravo, 2005). Por otra parte, las denuncias de la comunidad como veedores de los recursos naturales también se incrementan en la década de los noventa y la primera década del siglo XXI.

Teniendo en cuenta que una de las fases que genera mayor discusión 
por el conflicto con el ambiente es la prospección sísmica, el análisis de la información de las líneas realizadas en el área denominada "Corredor petrolero del Piedemonte llanero", muestra que durante la década de los setenta y los noventa se intensifico la sísmica en esta región. Para el gobierno nacional no resultaba claro cuál era la entidad encargada de la gestión ambiental de la actividad petrolera durante su auge en la década de los noventa. El país no tenía una institucionalidad ambiental lo suficientemente coordinada y fuerte para hacer frente al reto que ya presentaba tenaces consecuencias.

\section{A finales de esta década el Minis- terio de Salud y el Instituto Nacio- nal de Recursos Naturales Reno- vables y del Ambiente (Inderena) empezaron a trabajar en la gestión ambiental petrolera. Sin embargo, esto llevó a la conclusión de que la mayoría de las actividades ex- ploratorias, realizadas durante los años setenta y ochenta (por ejem- plo, los pozos perforados del año 1970 al año 1975, y otros más), no contaban con las licencias ni segui- mientos correspondientes, reflejan- do también la poca presencia del Estado en la entonces Intendencia de Casanare.}

Entre las principales afectaciones ambientales identificados en diferentes registros históricos, especialmente desde la década de los noventa en el corredor petrolero de Piedemonte, que han puesto en evidencia el conflicto directo del desarrollo de la actividad petrolera han sido: la adquisición de terrenos en el Piedemonte de la Cordillera Oriental en los municipios de Yopal, Aguazul y Tauramena; la pérdida de cobertura boscosa; los deslizamientos y desbordamientos de aguas residuales en el complejo Cusiana que generaron focos de contaminación; el cierre de pozos petroleros por incumplimiento de las empresas extranjeras con las licencias sanitarias de agua y aire; la contaminación de fuentes superficiales del río Cravo Sur y el río Meta; la muerte de fauna (insectos y aves) en el área de producción y en chimeneas (TEAS de Campos y estaciones de explotación de petróleo), entre otros.

Otros efectos que también deben ser parte del análisis integral del ambiente, son el incremento de obras y flujos de trabajadores, el gran incremento en la demanda de bienes y servicios, migraciones, inversiones económicas y regalías, la perdida de interés de la mano de 
obra del departamento en el sector agropecuario, la consolidación del corredor petrolero Pajarito-Cusiana-Cupiagua-Niscota, entre otros. Con el tiempo, estos municipios adquirieron una importancia estratégica regional en el sector económico de los hidrocarburos, buscando consolidar el centro facilitador de servicios básicos de la producción de petróleo en la Orinoquia.

Finalmente, al analizar los efectos ocasionados por el modelo petrolero frente a la dinámica de la gestión ambiental, es claro que el país no estaba preparado para asumir el reto institucional de administrar las áreas con presencia de petróleo y con una muy superior riqueza ambiental, para lograr un adecuado desarrollo.

\section{Conclusiones}

Las razones que han influido en la distribución espacial de los bloques de exploración y explotación de petróleo en Colombia y en el departamento de Casanare obedecen no solo a sus ventajas comparativas, sino también a decisiones geopolíticas y geoeconómicas nacionales e internacionales. En el escenario nacional se diseñaron políticas sectoriales para incre- mentar los recursos económicos del país con una visión de corto plazo, pretendiendo aprovechar la crisis del petróleo con precios de barril al alza, siempre bajo la premisa (desde 1970 al 2014) de que la intensificación de la exploración y explotación de petróleo estaban enfocadas a garantizar la denominada "autosuficiencia petrolera del país". En este sentido, a partir de los años noventa aumentó significativamente la dependencia de la economía local y nacional en la extracción de materias primas o commodities como el petróleo sin valor agregado. No obstante, a pesar de la intensificación de las campañas en la Orinoquia y especialmente en el Casanare, solo se logró un incremento considerable de la tasa de crecimiento en explotación de petróleo, pero no en el crecimiento positivo de las reservas probadas de petróleo. Esto indica que la búsqueda de nuevos yacimientos no obtuvo éxito pese a las metas de exploración definidas en estos períodos en los respectivos PND.

La existencia de recursos naturales como el petróleo es una ventaja comparativa para una región. Sin embargo, la exploración y explotación de este recurso genera serios efectos ambientales que crecen y 
se complican exponencialmente, sobre todo en las áreas que a priori tienen una historia de grandes conflictos sociales como el conflicto armado (que es el caso del Piedemonte llanero en el departamento de Casanare). Durante el período analizado, de la misma forma como se incrementaron las campañas de exploración y explotación de petróleo, también se agudizaron los enfrentamientos entre la fuerza pública y los grupos irregulares. De igual manera se intensificó la colonización en la franja de Piedemonte, aparecieron transnacionales de gran envergadura, y a su vez se triplicó la cantidad de población en estos municipios, especialmente en sus áreas urbanas. Además, la demanda de servicios públicos aumentó, entre otros efectos que indican una clara lucha por el dominio del territorio, lucha para la cual el gobierno en todas sus instancias no estaba preparado para dar respuesta.

Los efectos ambientales en estas zonas durante el período definido han sido consecuencia principalmente del modelo económico basado en la explotación de recursos naturales, y de la evolución e involución histórica en la formulación e implementación de las políticas sectoriales y ambientales en Colombia.
Aunque en diferentes análisis sobre la evolución histórica de la formulación e implementación de las políticas sectoriales y ambientales, especialmente para estas últimas, se tienden a clasificar en diferentes enfoques (Productivista, Conservacionista, Desarrollo Sostenible, Privatización de la gestión ambiental, entre otros), su implementación no se ha evidenciado de esa forma en el área de estudio. Su efecto no va más allá de la delimitación de áreas, numerosos planes de manejo y licencias, cargos legales a empresas operadoras, y tratamientos paliativos a problemas puntuales de contaminación ambiental. Esto se debe a que las políticas solo han tratado de dimensionar los conflictos de distribución ecológica sin explorar la complejidad de las relaciones sociales entre los actores locales, nacionales e internacionales, volviendolos instrumentos poco efectivos $y$ sin un dimensionamiento integral del ambiente. Por lo tanto, una gran parte de los conflictos son producto de la debilidad institucional y la falta de integración de las políticas ambientales y sectoriales. En aquellas áreas donde se desarrollan actividades de explotación de recursos naturales no renovables, una identificación de actores sociales muestra que 
son varios los intereses, entre ellos el dominio o control del territorio. En otras palabras, es indispensable cuestionar la dimensión territorial de las políticas.

Teniendo en cuenta el escenario actual de crisis por la caída del petróleo, la dependencia histórica (por más de treinta años) de este recurso por parte de los municipios del corredor petrolero de Piedemonte, y el posible escenario del posconflicto, es necesario reflexionar sobre los resultados de estas investigaciones como base para investigar sobre el futuro que más conviene a esta región, independientemente de la recuperación de los precios del petróleo. Será entonces necesario realizar un análisis del pasivo ambiental dejado en la región por las actividades de exploración y explotación de petróleo, con el fin de saber realmente qué tanto se conservaron los recursos y las características por las cuales el Piedemonte llanero se considera como una ecoregión estratégica. Además cuáles serían las opciones de un desarrollo sostenible que no implique necesariamente la expansión de una agresiva frontera agrícola en el Piedemonte, como ocurría durante la década de los setenta y los ochenta, a la luz de los actuales y subsecuentes lineamientos de los PND. Asimismo, es necesario realizar investigaciones sobre cómo territorializar las políticas, ya que sea cual sea la proyección que se realice para estos municipios, y en general para el departamento de Casanare, es necesario que desde el gobierno central se revisen, ajusten y propongan políticas de desarrollo con un enfoque territorial.

El análisis cronológico ya sea para la evaluación de políticas, los cambios en el territorio o la toma de decisiones, es indispensable para los análisis sobre ambiente que se realicen desde la óptica económica. En este sentido, es importante desde la geografía abordar temas como las denominadas tasas de descuento temporal y geográfico-espacial. Teniendo en cuenta trabajos como el de Perrings y Hannon (2001), quienes hacen referencia a que los tomadores de decisiones tienden a prescindir no sólo del tiempo sino también del espacio, el paso del tiempo tiene implicaciones para la equidad intergeneracional, enfocada a garantizar a las generaciones futuras el mismo potencial que han gozado las generaciones del presente. 


\section{Referencias}

ANH, Agencia Nacional de Hidrocarburos. (2014). Mapa de Tierras. Bogotá. Recuperado de http://www.anh.gov.co.

Aponte, E. (2006). La geohistoria, un enfoque para el estudio del espacio venezolano desde una perspectiva interdisciplinaria. Scripta Nova. Revista Electrónica de Geografía y Ciencias Sociales, 10(218). Recuperado de http://www.ub.edu/geocrit/sn/sn-218-08.htm

BP, British Petroleum Company. (2015). BP Statistical Review of World Energy. Oil sectionStatistical Review of World Energy [institutional report].

Bocco, G. y Urquijo, P. S. (2013). Geografía ambiental: reflexiones teóricas y práctica institucional Introducción. Región y Sociedad, 25(56), 75-101.

Bravo, E. (2005). Impacto de la actividad petrolera en las regiones tropicales [versión electrónica]. Quito: Oilwatch. Recuperado de http://www.oilwatch.org/doc/libros/maneraocci.pdf.

Bridge, G. (2008). Environmental Economic Geography: A Sympathetic Critique. Geoforum, 39(1), 76-81. Retrieved from http://doi.org/10.1016/j.geoforum.2007.06.005

Devia, C. (2011). Configuración territorial, petróleo y conflicto. Los casos Aguazul y Tauramena. 1974-2005 [tesis de maestría]. Bogotá, Convenio UPTC-IGAC. Recuperado de http:// biblioteca.igac.gov.co/janium/Documentos/tesisclaudia.pdf

Fontaine, G. (2004). Enfoques conceptuales y metodológicos para una sociología de los conflictos ambientales. Colombia: Friedrich-Ebert-Stiftung.

Guhl Nannetti, E. y Leyva, P. (2015). La gestión ambiental en Colombia, 1994-2014: ¿Un Esfuerzo Insostenible? Bogotá: Smurfit Kappa.

Heidkamp, C. P. (2008). A Theoretical Framework for a "Spatially Conscious" Economic Analysis of Environmental Issues. Geoforum, (39), 62-75. Retrieved from http://doi. $\operatorname{org} / 10.1016 /$ j.geoforum.2007.01.010

Perrings, C. y Hannon, B. (2001). An Introduction to Spatial Discounting. Journal of Regional Science, (41), 23-38. doi:10.1111/0022-4146.00205

Ramírez, M. y Yepes, J. M. (2011). Geopolítica de los recursos estratégicos: conflictos por agua en América latina. Revista de Relaciones Internacionales, Estrategia y Seguridad, 6(1), 149-165. Recuperado de http://www.scielo.org.co/scielo.php?pid=S190930632011000100008\&script=sci_arttext

Rebotier, J. (2013). Territorializar las políticas ambientales y de riesgo. XV Encontro da ANPUR, pp. $1-11$. 
Roth Deubel, A. N. (2004). Politicas públicas: formulación, implementación y evaluación. Bogotá: Ediciones Aurora.

Sabatini, F. (1997). Conflictos ambientales. Entre la globalización y la sociedad civil. Santiago: CIPMA.

Tobasura, I. (2006). La política ambiental en los Planes de Desarrollo en Colombia 1990-2006. Una visión crítica. Revista Luna Azul, (22), 8-19. Recuperado de http://vip.ucaldas.edu.co/ lunazul/downloads/Lunazul22_2.pdf

Trinca, D. (2010). La relación ambiente y desarrollo vista desde la geografía. Geotrópico, (4), $1-9$.

Universidad Nacional de Colombia. (2013). Observatorio de Procesos de Desarme, Desmovilización y Reintegración (ODDR). Estructuras de Autodefensas y Proceso de Paz en Colombia, Visibilización mediática DDR 'Caso Región Orinoquia’.

Velte, D. (2011). Las dinámicas de agotamiento de los recursos naturales y el papel de la prospectiva. Ekonomiaz, 76(1), 104-123.

Vitousek, P., D’Antonio, C., Loope, L., Rejmánek, M. y Westbrooks, R. (1997). Introduced Species: A Significant Component of Human-Caused Global Change. New Zealand Journal of Ecology, 21(1), 1-16. Retrieved from http://newzealandecology.org/system/files/articles/ NZJEcol21_1_1.pdf 
Recepción: 1 de junio de 2016

Evaluación: 10 de octubre de 2016

Aprobación: 14 de noviembre de 2016 